\title{
Universal Wind Profile for Conventionally Neutral Atmospheric Boundary Layers
}

\author{
Luoqin Liu $\odot$, Srinidhi N. Gadde $\odot$, and Richard J. A. M. Stevens $\odot^{\dagger}$ \\ Physics of Fluids Group, Max Planck Center Twente for Complex Fluid Dynamics, University of Twente, \\ 7500 AE Enschede, Netherlands
}

(Received 26 August 2020; revised 28 October 2020; accepted 28 January 2021; published 9 March 2021)

\begin{abstract}
Conventionally neutral atmospheric boundary layers (CNBLs), which are characterized with zero surface potential temperature flux and capped by an inversion of potential temperature, are frequently encountered in nature. Therefore, predicting the wind speed profiles of CNBLs is relevant for weather forecasting, climate modeling, and wind energy applications. However, previous attempts to predict the velocity profiles in CNBLs have had limited success due to the complicated interplay between buoyancy, shear, and Coriolis effects. Here, we utilize ideas from the classical Monin-Obukhov similarity theory in combination with a local scaling hypothesis to derive an analytic expression for the stability correction function $\psi=-c_{\psi}(z / L)^{1 / 2}$, where $c_{\psi}=4.2$ is an empirical constant, $z$ is the height above ground, and $L$ is the local Obukhov length based on potential temperature flux at that height, for CNBLs. An analytic expression for this flux is also derived using dimensional analysis and a perturbation method approach. We find that the derived profile agrees excellently with the velocity profile in the entire boundary layer obtained from high-fidelity large eddy simulations of typical CNBLs.
\end{abstract}

DOI: 10.1103/PhysRevLett.126.104502

Introduction.-For well over a century, wall-bounded turbulent flows have been studied extensively [1]. A focus area is the derivation and characterization of the mean velocity profile. In 1925, Prandtl [2] recognized that the velocity profile in the inertial sublayer is approximately logarithmic based on his mixing length hypothesis. In the 1930s, von Kármán [3,4] derived the logarithmic law of the wall analytically using dimensional analysis. In 1956, Coles [5] showed using measurement data that the velocity profile can be described more accurately via the sum of the logarithmic law and a wake function. Since then, the law of the wall has been the pillar of the description of wallbounded turbulence [6-8]. Recently, the universality of the law of the wall has been supported by theoretical and experimental studies [9-11].

The dynamics in atmospheric boundary layers, where most human activity and biological processes occur, are much more complicated as turbulence is generated by shear stress and buoyancy [12], while the Coriolis force creates a wind veer [13]. In 1954, Monin and Obukhov [14] introduced a stability correction function $\psi$ to account for deviations to the logarithmic wind speed profile caused by thermal stratification. Based on the Buckingham $\Pi$ theorem, Monin and Obukhov concluded that $\psi$ is only a function of the atmospheric stability parameter $z / L_{w}$, where $z$ is the vertical height above the ground and $L_{w}$ is the Obukhov length based on the surface potential temperature flux. The universality of this well-known Monin-Obukhov similarity theory (MOST) [14-16] has been established in the surface layers of stable and convective atmospheric boundary layers in many field experiments [17], as well as large eddy simulations (LESs) [18]. Therefore, the MOST is nowadays regarded as the starting point of modern micrometeorology [17].

Conventionally neutral atmospheric boundary layers (CNBLs) are also frequently observed and are often considered in fundamental studies [19-26]. In contrast to stable and convective atmospheric boundary layers, CNBLs are characterized with zero surface potential temperature flux and capped by an inversion of potential temperature. However, the classical MOST is not applicable to CNBLs, because the surface potential temperature flux is zero, due to which $L_{w}$ is no longer a relevant scale [18]. Many studies [23,27-35] have tried to predict the velocity profile in CNBLs, but so far with limited success since none of them have considered the effect of potential temperature flux near the capping inversion layer. Therefore, the logarithmic law of the wall without stability correction is still commonly used to predict the wind speed profile in CNBLs [36-40]. However, an analytical description of the velocity profiles in CNBLs is of great fundamental interest and relevant for meteorological applications $[12,35]$.

Theory.-In this Letter, we derive the potential temperature flux profile using dimensional analysis and a perturbation method approach. To account for the deviation to the logarithmic wind speed profile, we use ideas from the MOST in combination with a local similarity hypothesis. Therefore, we introduce a stability correction function $\psi$ that depends only on the local stability parameter $z / L$, where $L$ is the local Obukhov length based on the local potential temperature flux. The canonical shape of $\psi$ is 
determined by asymptotic analysis. The derived universality profiles for the potential temperature flux and the wind speed profiles are confirmed by the excellent agreements with the results of high-fidelity LESs.

Based on the dimensional analysis (a derivation can be found in Sec. III of the Supplemental Material [41]), the potential temperature flux can be written as

$$
\frac{\beta z_{0} q}{u_{*}^{3}}=-\Pi(\mathrm{Ro}, \mathrm{Zi}, \xi)=-\mathrm{Ro}^{r} \mathrm{Zi}^{s} \Pi_{1}(\xi) .
$$

Here $q$ is the potential temperature flux, $u_{*}$ is the friction velocity, $\beta$ is the buoyancy parameter, $\Pi$ and $\Pi_{1}$ are dimensionless functions, Ro $=u_{*} /\left(|f| z_{0}\right)$ is the Rossby number, with $f$ as the Coriolis parameter and $z_{0}$ as the roughness height, $\mathrm{Zi}=N /|f|$ is the Zilitinkevich number [28], with $N$ as the free-atmosphere Brunt-Väisälä frequency, and $r$ and $s$ are the power exponents for Ro and $\mathrm{Zi}$, respectively. The dimensionless parameter $\xi=z / h^{\prime}$, where $h^{\prime}=h /\left(1-0.05^{2 / 3}\right)$ and $h$ is the boundary layer height at which the total momentum flux reaches $5 \%$ of the surface value. This conventional definition underestimates the actual boundary layer height. The functional form of $h^{\prime}$ follows from the fact that the dimensionless total momentum flux follows a power law with exponent $3 / 2$ [42]. We note that the first equality in Eq. (1) is unconditionally valid, although the corresponding functional form is hard to determine analytically. The second equality is valid under the presumed power-law dependence of $\Pi$ on the two independent dimensionless parameters $\mathrm{Ro}$ and $\mathrm{Zi}$. The values of $s$ and $r$ will be determined later from our high-fidelity LES data.

We emphasize that other definitions for the boundary layer height, which are based on, for example, the vertical wind speed or potential temperature profiles, are also commonly used $[25,31,35]$. In particular, the boundary layer height $h_{t}$ is defined as the height at which the potential temperature flux reaches its minimum value. Previous studies [25,35,43-45] showed that the potential temperature flux in CNBLs decreases linearly from zero at the surface to a minimum value at $z=h_{t}$ and then increases to zero for $z \geq h^{\prime}$. As explained in Sec. IV of the Supplemental Material [41], the ratio $h_{t} / h^{\prime} \equiv 1-2 \epsilon$ is a function of $\mathrm{Zi}$. However, as we will see later, the dependence of $\epsilon$ on $\mathrm{Zi}$ is limited over the parameter regime under consideration. Clearly, $\epsilon \ll 1$ represents the half-capping layer thickness normalized by the height $h^{\prime}$, where the potential temperature flux recovers steeply to zero. Therefore, we propose the following ordinary differential equation to model the potential temperature flux:

$$
-\epsilon \Pi_{1}^{\prime \prime}+\Pi_{1}^{\prime}=c_{\Pi}, \quad \Pi_{1}(0)=\Pi_{1}(1)=0 .
$$

Here $c_{\Pi}$ is the slope of the dimensionless total potential temperature flux $-\left(\beta z_{0} q\right) /\left(u_{*}^{3} \mathrm{Zi}^{s} \mathrm{Ro}^{r}\right)$ in the surface layers, which can be determined from simulation or measurement data. The solution of $\Pi_{1}$ reads

$$
\Pi_{1}= \begin{cases}c_{\Pi}\left(\xi-\frac{e^{\xi / \epsilon}-1}{e^{1 / \epsilon}-1}\right), & \xi \leq 1, \\ 0, & \xi>1 .\end{cases}
$$

Note that Eq. (2) and its solution Eq. (3) is reminiscent of the classical singular perturbation method [46]: The outer solution (close to the wall) is a linear function of $\xi$ and the inner solution (close to the capping inversion layer) is controlled by a small parameter $\epsilon$.

In contrast to the classical MOST [14] where the normalized wind speed gradient is assumed to be a universal function, we introduce a stability correction function $\psi$ to account for the deviation of the logarithmic profile. Therefore, we write the wind speed profile as

$$
\frac{\kappa U_{\mathrm{mag}}}{u_{*}}=\ln \left(\frac{z}{z_{0}}\right)-\psi\left(\frac{z}{L}\right),
$$

where $\kappa=0.4$ is the von Kármán constant, $U_{\text {mag }}$ is the mean wind speed, and $\psi$ is the stability correction function that depends only on the dimensionless stability parameter $z / L$. According to the local scaling hypothesis $[42,47], L$ is defined as the local Obukhov length

$$
\frac{1}{L} \equiv-\frac{\kappa \beta q}{u_{*}^{3}} .
$$

It is worth pointing out that the dimensionless slope $\left(\kappa z / u_{*}\right) d U_{\text {mag }} / d z$ is usually regarded as a universal function of the stability parameter $z / L$ in the stable and convective atmospheric boundary layers [48]. However, under the assumption of Eq. (4), this slope is no longer a universal function of $z / L$.

To determine the canonical shape of $\psi$, we assume

$$
\psi=-c_{\psi}\left(\frac{z}{L}\right)^{p}
$$

where $p$ is the power exponent to be determined analytically below, and $c_{\psi}$ is an empirical constant. Recall that very close to the wall [see Eq. (3)]

$$
\Pi_{1} \rightarrow c_{\Pi} \xi=c_{\Pi} \frac{z}{h^{\prime}} \quad \text { as } \frac{z}{h^{\prime}} \rightarrow 0 .
$$

Then, from asymptotic analysis [49], we find that

$$
\frac{z}{L}=\frac{\kappa z}{z_{0}} \Pi \rightarrow \frac{c_{\Pi} \kappa h^{\prime}}{z_{0}} \mathrm{Zi}^{s} \operatorname{Ro}^{r}\left(\frac{z}{h^{\prime}}\right)^{2} \text { as } \frac{z}{h^{\prime}} \rightarrow 0 .
$$

Zilitinkevich and Esau [23] showed that in the surface layers of stable, truly neutral, and conventionally neutral atmospheric boundary layers $\psi=-C_{u} z / L_{M}$. Here $C_{u}$ is a 
TABLE I. Summary of all simulated cases, where the Zi and Ro range covers the values found in typical CNBLs at mid to high latitudes $[58,59]$.

\begin{tabular}{lcccccc}
\hline \hline Case no. & $A$ & $B$ & $C$ & $D$ & $E$ & $F$ \\
\hline $\mathrm{Zi}$ & 51.2 & 88.7 & 88.7 & 88.7 & 88.7 & 153.6 \\
$\mathrm{Ro}$ & $2.7 \times 10^{7}$ & $4.5 \times 10^{4}$ & $3.7 \times 10^{5}$ & $3.2 \times 10^{6}$ & $2.7 \times 10^{7}$ & $2.7 \times 10^{7}$ \\
$\epsilon$ & 0.1186 & 0.1148 & 0.1191 & 0.1213 & 0.1224 & 0.1341 \\
$c_{\Pi}$ & 0.0335 & 0.0335 & 0.0330 & 0.0329 & 0.0330 & 0.0336 \\
\hline \hline
\end{tabular}

dimensionless constant and $L_{M}$ is the combined turbulent length scale, which in CNBLs can be estimated as $|f| L_{M} / u_{*}=\left(1+C_{m}^{2} \mathrm{Zi}^{2}\right)^{-1 / 2}$, where $C_{m}$ is an empirical constant ([23], see also the Supplemental Material [41]). To match with the result of Zilitinkevich and Esau [23] in the surface layer, we find that $p=1 / 2$. Clearly, the determination of $p$ is independent of the values of $c_{\Pi}, c_{\psi}, r, s$, and $\epsilon$. Thus, the wind speed profile is given by

$$
\frac{\kappa U_{\mathrm{mag}}}{u_{*}}= \begin{cases}\ln \left(\frac{z}{z_{0}}\right)+c_{\psi}\left(\frac{z}{L}\right)^{1 / 2}, & \xi \leq \xi_{0}, \\ \frac{\kappa G}{u_{*}}, & \xi>\xi_{0} .\end{cases}
$$

Here $G$ is the geostrophic wind speed, $\xi_{0}$ is the highest intersection point of the curves described by the upper and lower expressions in Eq. (9), $z / L$ is the dimensionless stability parameter predicted by the potential temperature flux model [i.e., Eqs. (1) and (3)], and $c_{\psi}$ is the empirical constant that can be determined from simulation or measurement data.

Validation.-To verify the universality of the wind speed profile for CNBLs, we perform six high-fidelity LESs. In the simulations, a CNBL over a flat surface with periodic conditions in horizontal directions is considered. The flow is initialized with uniform geostrophic wind speed and a linear potential temperature profile with a constant gradient $[35,44]$. The simulations are performed with an in-house code [50-55], which employs a pseudospectral discretization in the horizontal directions and a second-order finite difference method in the vertical direction. We employ the advanced anisotropic minimum dissipation model to parametrize the subgrid scale shear stress and potential temperature flux [56]. The horizontal domain size is more than 6 times larger than the boundary layer height, and the grid resolution is $288^{3}$. We ensure that all simulations have reached the quasistationary state and the statistics are averaged over one inertial period [57]. A summary of all simulated cases is presented in Table I. The simulated Zilitinkevich number $\mathrm{Zi}$ and Rossby number Ro range covers the values found in typical CNBLs at mid to high latitudes [58,59]. More details about the numerical method and simulation setup can be found in the Supplemental Material [41].

Figure 1 shows the vertical profile of the dimensionless mean total momentum flux $\tau / \tau_{w}$, where $\tau$ is the total momentum flux and $\tau_{w}$ is its surface value. All different cases in Table I are shown in the figure (filled symbols). Nieuwstadt [42] analytically determined that the total momentum flux profile in stable atmospheric boundary layers scales as $\tau / \tau_{w}=(1-z / h)^{3 / 2}$. In Fig. 1 we show that this expression is still valid for CNBLs when we consider the previously introduced boundary layer thickness $h^{\prime}=h /\left(1-0.05^{2 / 3}\right)$. The finding that the dimensionless momentum flux profiles obtained from all LESs collapse to the theoretical curve (see Fig. 1) confirms that $h^{\prime}$ is the appropriate boundary layer height scale to consider.

To determine the values of the power indices $r$ and $s$, we take the vertical derivative of Eq. (1); see details in Sec. III of the Supplemental Material [41]. Figure 2 shows the dimensionless mean potential temperature gradient $\ln \left(-\beta z_{0} q^{\prime} / u_{*}^{3}\right)$ versus (a) the Rossby number $\ln$ Ro and (b) the Zilitinkevich number $\ln \mathrm{Zi}$ in the surface layer, where $q^{\prime}=d q / d \xi$. The slopes of the curve shown in the figure determine the values of the power exponents $r$ and $s$. In the parameter regime under consideration $r=-1.002 \approx$ -1 and $s=1.004 \approx 1$ describe the data very well. We note that the values of $c_{\Pi}$ can also be determined from the figure and the results are listed in Table I.

Figure 3 shows the vertical profile of the dimensionless mean potential temperature flux $q /|q|_{\max }$, which reduces to almost zero at $z / h^{\prime} \geq 1$. The potential temperature flux first decreases linearly from zero at the surface to a minimum value at $z=h_{t} \equiv(1-2 \epsilon) h^{\prime}$, and then increases rapidly to zero in a narrow region $\left(1-2 \epsilon \leq z / h^{\prime} \leq 1\right)$ since $\epsilon \ll 1$.

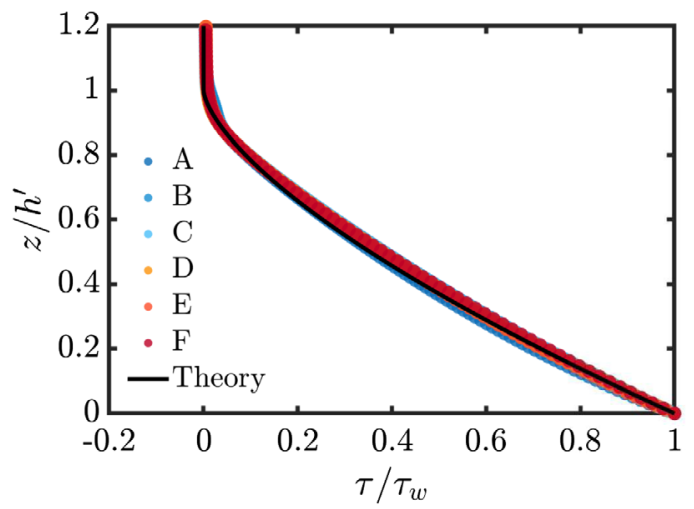

FIG. 1. Vertical profile of dimensionless mean total momentum flux $\tau / \tau_{w}$. Filled symbols, LES data; solid line, theoretical curve given by $\tau / \tau_{w}=\left(1-z / h^{\prime}\right)^{3 / 2}$. 

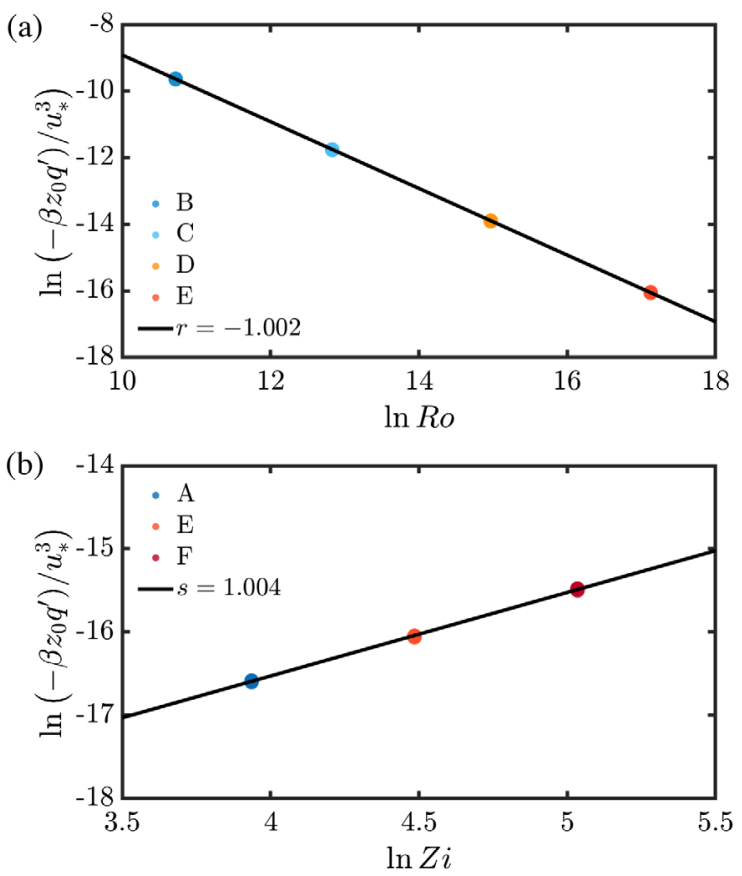

FIG. 2. Dimensionless mean potential temperature gradient in the surface layer versus (a) the Rossby number Ro and (b) the Zilitinkevich number $\mathrm{Zi}$, where $q^{\prime}=d q / d \xi$. The values of the slope are (a) $r=-1.002 \approx-1$ and (b) $s=1.004 \approx 1$, which are determined by a least-squares fitting procedure.

The value of $\epsilon$ is expected to depend only on $\mathrm{Zi}$ (see the Supplemental Material [41]). The data in Table I show that for the parameter range under consideration the variation of $\epsilon$ is limited, and therefore, we take $\epsilon=0.12$ to describe the

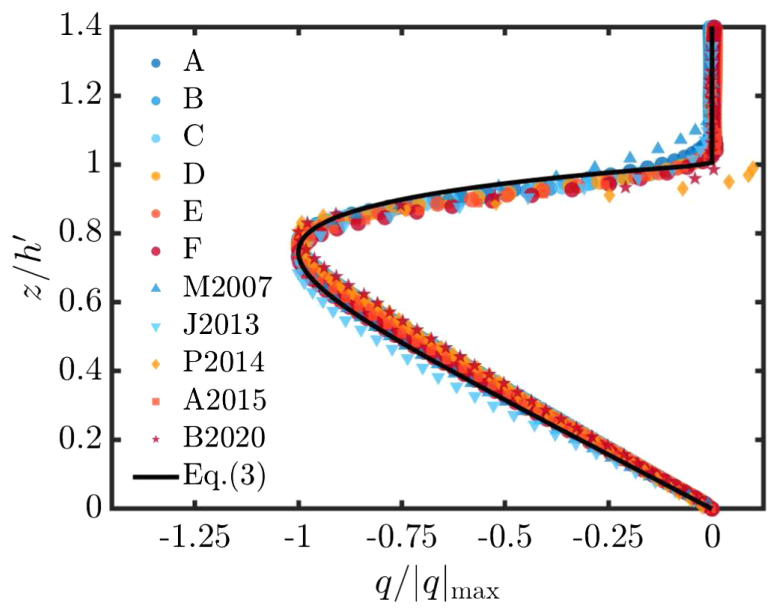

FIG. 3. Vertical profile of dimensionless mean potential temperature flux $q /|q|_{\max }$. Filled circles, LES data of the present Letter; filled triangles, prediction based on a turbulence closure given by Mauritsen et al. [43]; filled inverted triangles, DNS data of Jonker et al. [60]; filled diamonds, LES data of Pedersen et al. [44]; filled squares, LES data of Allaerts and Meyers [25]; filled stars, LES data of Berg et al. [45]; solid line, theoretical prediction given by Eq. (3) with $\epsilon=0.12$. data here. Evidently, all LES data of the present Letter (filled symbols) collapse very well to the introduced theoretical model (solid line), which validates the chosen approach. For comparison, the prediction based on a turbulence closure given by Mauritsen et al. [43], the direct numerical simulations (DNS) data performed by Jonker et al. [60], and the LES data taken from Pedersen et al. [44], Allaerts and Meyers [25], and Berg et al. [45] are also shown in the figure. The overall agreement between the theoretical prediction and the data from previous studies $[25,43-45,60]$ is very good, which confirms the universality of the proposed potential temperature flux profile.

Figure 4 shows the vertical profile of the dimensionless wind speed for two typical cases, which covers the $\mathrm{Zi}$ and Ro number range of typical CNBLs at mid to high latitudes $[58,59]$. The filled symbols are LES data, the dashed line is the theoretical prediction given by the logarithmic law, the blue line is the prediction of Zilitinkevich and Esau [23], the yellow line is the prediction of Gryning et al. [29], the red line is the prediction of Kelly et al. [35], and the black line is the prediction given by Eq. (9) with $c_{\psi}=4.2$, where the potential temperature flux profile is modeled by Eq. (3)
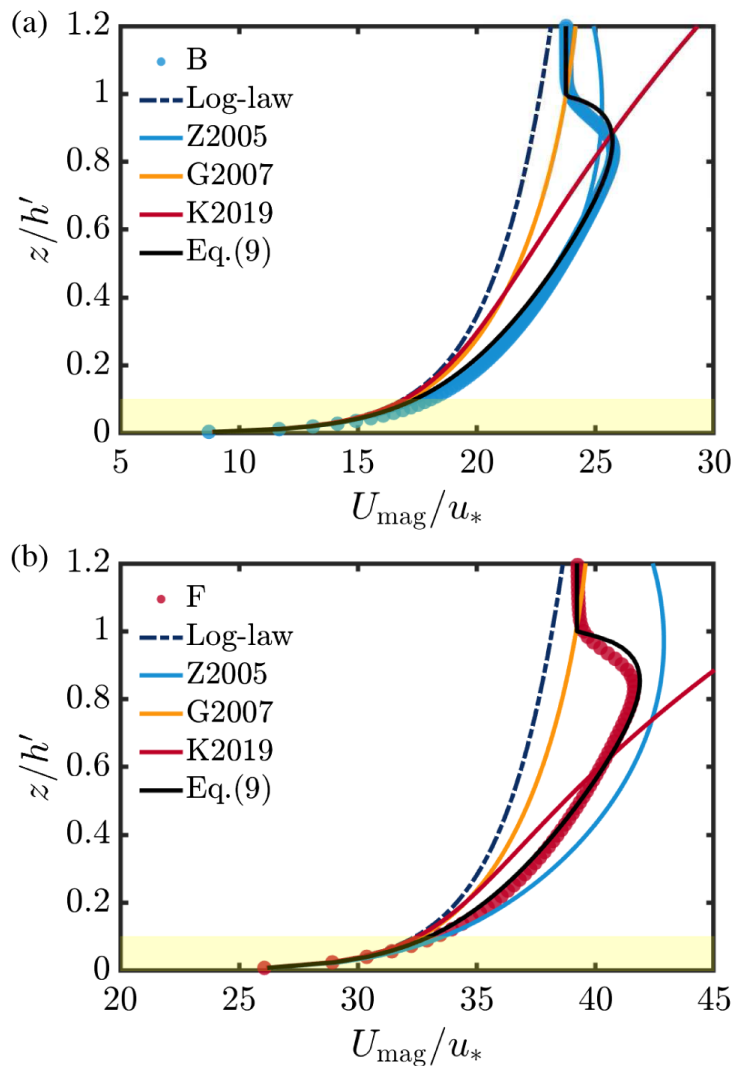

FIG. 4. Vertical profile of mean wind speed for (a) case $B$ and (b) case $F$. Filled symbols, LES data; dashed line, prediction given by the logarithmic law; blue line, prediction given by Zilitinkevich and Esau [23]; yellow line, prediction given by Gryning et al. [29]; red line, prediction given by Kelly et al. [35]; black line, prediction given by Eq. (9) with $c_{\psi}=4.2$. 
with $c_{\Pi}=0.0332$ and $\epsilon=0.12$ (see Table I). The empirical constant $c_{\psi}$ is determined such that it can predict the wind speed profiles of all cases in Table I with minimum discrepancies. The figure shows that the logarithmic law only accurately captures the wind speed in the lower $10 \%$ of the boundary layer, also known as the surface layer (shaded region). The theory given by Gryning et al. [29] focuses on capturing the wind speed at the top of the CNBL, but does not capture the effect of the low-level jet. The theory given by Kelly et al. [35] is focused on the lower part of the CNBL. The predictions by Zilitinkevich and Esau [23] agree well with the LES data in the lower part of the CNBL, but do not capture the low-level jet, which is represented in our approach. In contrast, the agreement between the proposed profile (9) and the LES data is nearly perfect in the entire boundary layer and much better than all previous approaches. This excellent agreement confirms the universality of our proposed wind profile (9) in the considered parameter range of CNBLs.

Summary.-We propose a universal velocity profile for CNBL derived using a local similarity hypothesis combined with ideas from the classical Monin-Obukhov similarity theory. We introduce a stability correction function $\psi$ to account for the deviation of the logarithmic law. The canonical shape of $\psi$ is determined theoretically as $\psi=-c_{\psi}(z / L)^{1 / 2}$, where $c_{\psi}=4.2$ is an empirical constant determined from simulation data, $z$ is the vertical height above the surface, and $L$ is the local Obukhov length. An analytical expression for the potential temperature flux profile is also derived from dimensional analysis and perturbation method. The universality of the proposed profile (9) has been confirmed by its excellent agreement with high-fidelity LES results for $\mathrm{Ro}=\left[4.5 \times 10^{4}\right.$, $\left.2.7 \times 10^{7}\right]$ and $\mathrm{Zi} \in[51,154]$, where the $\mathrm{Zi}$ and Ro number range covers the range of values observed in typical CNBLs at mid to high latitudes. Further work is required to assess the applicability of the approach to other parameter regimes.

We appreciate very much the valuable comments of the anonymous referees. We acknowledge Dr. K. L. Chong and Dr. Y.X. Li for insightful discussion. This work is part of the Shell-NWO/FOM-initiative Computational sciences for energy research of Shell and Chemical Sciences, Earth and Live Sciences, Physical Sciences, Stichting voor Fundamenteel Onderzoek der Materie (FOM) and STW, and an STW VIDI Grant (No. 14868). This work was carried out on the national e-infrastructure of SURFsara, a subsidiary of SURF cooperation, the collaborative ICT organization for Dutch education and research.

*luoqin.liu@utwente.nl

†r.j.a.m.stevens@utwente.nl
[1] A. J. Smits, B. J. McKeon, and I. Marusic, High Reynolds number wall turbulence, Annu. Rev. Fluid Mech. 43, 353 (2011).

[2] L. Prandtl, Bericht über Untersuchungen zur ausgebildeten Turbulenz, Z. Angew. Math. Mech. 5, 136 (1925).

[3] T. von Kármán, Mechanische Ähnlichkeit und Turbulenz, Nachr. Ges. Wiss. Göttingen Math. Phys. Klasse 5, 58 (1930).

[4] T. von Kármán, Mechanische Ähnlichkeit und Turbulenz, Proc. Third Internat. Congr. Appl. Mech. Stockholm 1, 85 (1931).

[5] D. Coles, The law of the wake in the turbulent boundary layer, J. Fluid Mech. 1, 191 (1956).

[6] H. Tennekes and J. L. Lumley, A First Course in Turbulence (The MIT Press, Cambridge, Massachusetts, 1972).

[7] S. B. Pope, Turbulent Flows (Cambridge University Press, Cambridge, England, 2000).

[8] P. Davidson, Turbulence: An Introduction for Scientists and Engineers (Oxford University Press, Oxford, 2004).

[9] I. Marusic, J. P. Monty, M. Hultmark, and A. J. Smits, On the logarithmic region in wall turbulence, J. Fluid Mech. 716, R3 (2013).

[10] P. Luchini, Universality of the Turbulent Velocity Profile, Phys. Rev. Lett. 118, 224501 (2017).

[11] M. Samie, I. Marusic, N. Hutchins, M. K. Fu, Y. Fan, M. Hultmark, and A. J. Smits, Fully resolved measurements of turbulent boundary layer flows up to $R e_{\tau}=20000$, J. Fluid Mech. 851, 391 (2018).

[12] G. G. Katul, A. G. Konings, and A. Porporato, Mean Velocity Profile in a Sheared and Thermally Stratified Atmospheric Boundary Layer, Phys. Rev. Lett. 107, 268502 (2011).

[13] M. F. Howland, A. S. Ghate, and S. K. Lele, Influence of the geostrophic wind direction on the atmospheric boundary layer flow, J. Fluid Mech. 883, A39 (2020).

[14] A. S. Monin and A. M. Obukhov, Basic laws of turbulent mixing in the surface layer of the atmosphere, Tr. Akad. Nauk SSSR Geophiz. Inst. 24, 163 (1954).

[15] A. M. Obukhov, Turbulence in an atmosphere with inhomogeneous temperature, Trans. Inst. Teoret. Geoz. Akad. Nauk SSSR 1, 95 (1946).

[16] A. S. Monin and A. M. Yaglom, Statistical Fluid Mechanics, Vol. 1. Mechanics of Turbulence (The MIT Press, Cambridge, Massachusetts, 1971).

[17] T. Foken, 50 years of the Monin-Obukhov similarity theory, Boundary-Layer Meteorol. 119, 431 (2006).

[18] S. Khanna and J. G. Brasseur, Analysis of Monin-Obukhov similarity from large-eddy simulation, J. Fluid Mech. 345, 251 (1997).

[19] R. A. Brost, D. H. Lenschow, and J. C. Wyngaard, Marine stratocumulus layers. Part 1: Mean conditions, J. Atmos. Sci. 39, 800 (1982).

[20] A. L. M. Grant, Observations of boundary layer structure made during the 1981 KONTUR experiment, Q. J. R. Meteorol. Soc. 112, 825 (1986).

[21] M. Tjernström and A.-S. Smedman, The vertical turbulence structure of the coastal marine atmospheric boundary layer, J. Geophys. Res.: Oceans 98, 4809 (1993).

[22] S. S. Zilitinkevich and I. N. Esau, On integral measures of the neutral barotropic planetary boundary layer, BoundaryLayer Meteorol. 104, 371 (2002). 
[23] S. S. Zilitinkevich and I. N. Esau, Resistance and heattransfer laws for stable and neutral planetary boundary layers: Old theory advanced and re-evaluated, Q. J. R. Meteorol. Soc. 131, 1863 (2005).

[24] S. S. Zilitinkevich, I. Esau, and A. Baklanov, Further comments on the equilibrium height of neutral and stable planetary boundary layers, Q. J. R. Meteorol. Soc. 133, 265 (2007).

[25] D. Allaerts and J. Meyers, Large eddy simulation of a large wind-turbine array in a conventionally neutral atmospheric boundary layer, Phys. Fluids 27, 065108 (2015).

[26] D. Allaerts and J. Meyers, Boundary-layer development and gravity waves in conventionally neutral wind farms, J. Fluid Mech. 814, 95 (2017).

[27] S. S. Zilitinkevich, V. L. Perov, and J. C. King, Near-surface turbulent fluxes in stable stratification: Calculation for use in general circulation models, Q. J. R. Meteorol. Soc. 128, 1571 (2002).

[28] I. N. Esau, Parameterization of a surface drag coefficient in conventionally neutral planetary boundary layer, Ann. Geophys. 22, 3353 (2004).

[29] S.-E. Gryning, E. Batchvarova, B. Brümmer, H. Jorgensen, and S. Larsen, On the extension of the wind profile over homogeneous terrain beyond the surface boundary layer, Boundary-Layer Meteorol. 124, 251 (2007).

[30] M. Kelly and S.-E. Gryning, Long-term mean wind profiles based on similarity theory, Boundary-Layer Meteorol. 136, 377 (2010).

[31] M. Abkar and F. Porté-Agel, The effect of free-atmosphere stratification on boundary-layer flow and power output from very large wind farms, Energies 6, 2338 (2013).

[32] M. Optis, A. Monahan, and F. C. Bosveld, Moving beyond Monin-Obukhov similarity theory in modelling wind-speed profiles in the lower atmospheric boundary layer under stable stratification, Boundary-Layer Meteorol. 153, 497 (2014).

[33] M. Kelly and I. Troen, Probabilistic stability and "tall" wind profiles: Theory and method for use in wind resource assessment, Wind Energy 19, 227 (2016).

[34] Q. F. Jiang, S. P. Wang, and P. Sullivan, Large-eddy simulation study of log laws in a neutral Ekman boundary layer, J. Atmos. Sci. 75, 1873 (2018).

[35] M. Kelly, R. A. Cersosimo, and J. Berg, A universal wind profile for the inversion-capped neutral atmospheric boundary layer, Q. J. R. Meteorol. Soc. 145, 982 (2019).

[36] C. G. Rossby and R. B. Montgomery, The layers of frictional influence in wind and ocean currents, Pap. Phys. Oceanogr. Meteor. 3, 1 (1935).

[37] A. K. Blackadar, The vertical distribution of wind and turbulent exchange in a neutral atmosphere, J. Geophys. Res. 67, 3095 (1962).

[38] A. K. Blackadar and H. Tennekes, Asymptotic similarity in neutral barotropic planetary boundary layers, J. Atmos. Sci. 25, 1015 (1968).

[39] H. Tennekes, The logarithmic wind profile, J. Atmos. Sci. 30, 234 (1973).

[40] M. Horiguchi, T. Hayashi, A. Adachi, and S. Onogi, Largescale turbulence structures and their contributions to the momentum flux and turbulence in the near-neutral atmospheric boundary layer observed from a 213-m tall meteorological tower, Boundary-Layer Meteorol. 144, 179 (2012).

[41] See the Supplemental Material at http://link.aps.org/ supplemental/10.1103/PhysRevLett.126.104502 which includes additional information on numerical simulations, derivation of the potential temperature flux, formulations of existing wind speed models, and Refs. [1,2,5,7,8].

[42] F. T. M. Nieuwstadt, The turbulent structure of the stable, nocturnal boundary layer, J. Atmos. Sci. 41, 2202 (1984).

[43] T. Mauritsen, G. Svensson, S. S. Zilitinkevich, I. N. Esau, L. Enger, and B. Grisogono, A total turbulent energy closure model for neutrally and stably stratified atmospheric boundary layers, J. Atmos. Sci. 64, 4113 (2007).

[44] J. G. Pedersen, S.-E. Gryning, and M. Kelly, On the structure and adjustment of inversion-capped neutral atmospheric boundary-layer flows: Large-eddy simulation study, Boundary-Layer Meteorol. 153, 43 (2014).

[45] J. Berg, E. G. Patton, and P. P. Sullivan, Large-eddy simulation of conditionally neutral boundary layers: A mesh resolution sensitivity study, J. Atmos. Sci. 77, 1969 (2020).

[46] M. V. Dyke, Perturbation Methods in Fluid Mechanics (Parabolic Press, Stanford, California, 1975).

[47] Z. Sorbjan, On similarity in the atmospheric boundary layer, Boundary-Layer Meteorol. 34, 377 (1986).

[48] J. A. Businger, J. C. Wyngaard, Y. Izumi, and E. F. Bradley, Flux-profile relationships in the atmospheric surface layer, J. Atmos. Sci. 28, 181 (1971).

[49] C. C. Lin and L. A. Segel, Mathematics Applied to Deterministic Problems in the Natural Sciences (Society for Industrial and Applied Mathematics, Philadelphia, 1988).

[50] E. Bou-Zeid, C. Meneveau, and M. B. Parlange, A scaledependent Lagrangian dynamic model for large eddy simulation of complex turbulent flows, Phys. Fluids 17, 025105 (2005).

[51] R. J. A. M. Stevens, M. Wilczek, and C. Meneveau, Large eddy simulation study of the logarithmic law for high-order moments in turbulent boundary layers, J. Fluid Mech. 757, 888 (2014).

[52] S. N. Gadde and R. J. A. M. Stevens, Effect of Coriolis force on a wind farm wake, J. Phys. Conf. Ser. 1256, 012026 (2019).

[53] S. N. Gadde, A. Stieren, and R. J. A. M. Stevens, Large eddy simulations of stratified atmospheric boundary layers: Comparison of different sub-grid models, Boundary-Layer Meteorol. (2020), https://doi.org/10.1007/s10546-02000570-5.

[54] L. Liu and R. J. A. M. Stevens, Effects of two-dimensional steep hills on the performance of wind turbines and wind farms, Boundary-Layer Meteorol. 174, 61 (2020).

[55] L. Liu, S. N. Gadde, and R. J. A. M. Stevens, Geostrophic drag law for conventionally neutral atmospheric boundary layers revisited, Q. J. R. Meteorol. Soc. 1 (2020).

[56] M. Abkar and P. Moin, Large eddy simulation of thermally stratified atmospheric boundary layer flow using a minimum dissipation model, Boundary-Layer Meteorol. 165, 405 (2017). 
[57] G. N. Coleman, J. H. Ferziger, and P. R. Spalart, Direct simulation of the stably stratified turbulent Ekman layer, J. Fluid Mech. 244, 677 (1992).

[58] G. D. Hess and J.R. Garratt, Evaluating models of the neutral, barotropic planetary boundary layer using integral measures: Part I. Overview, Boundary-Layer Meteorol. 104, 333 (2002).
[59] S. S. Zilitinkevich, S. A. Tyuryakov, Y. I. Troitskaya, and E. A. Mareev, Theoretical models of the height of the atmospheric boundary layer and turbulent entrainment at its upper boundary, Izv. Atmos. Oceanic Phys. 48, 133 (2012).

[60] H. J. J. Jonker, M. van Reeuwijk, P. P. Sullivan, and E. G. Patton, On the scaling of shear-driven entrainment: A DNS study, J. Fluid Mech. 732, 150 (2013). 